\title{
The feasibility of vagus nerve preservation and double-flap technique in laparoscopic proximal gastrectomy
}

\author{
Ke-kang SUN ${ }^{1}$, Zhen WANG ${ }^{1}$, Wei PENG ${ }^{1}$, Ming CHENG ${ }^{1}$, zheng-rong $\mathrm{CHEN}^{1}$, rui \\ $\mathrm{REN}^{1}$, qiang $\mathrm{CHEN}^{1}$, and Yong-you WU \\ ${ }^{1}$ Affiliation not available
}

May 8, 2021

\begin{abstract}
Background: Laparoscopic proximal gastrectomy with an anti-reflux procedure, as a function-preserving surgery, has been a common treatment strategy for proximal early gastric cancer. This study aimed to develop a new surgical technique for these patients. Methods: Laparoscopic proximal gastrectomy with vagus nerve preservation and double-flap technique was performed in patients who could retain two-thirds of the stomach. The hepatic branches and the celiac branches were both preserved. A seromuscular double-flap was created through the auxiliary incision, and the anastomosis was performed under laparoscopy. The detailed surgical procedure and preliminary results were presented. Results: Six patients underwent this procedure with no conversion to open surgery. No intraoperative and postoperative complications occurred during the perioperative period. No patients showed diarrhea and appetite loss, and body weight regained preoperative level 6 month later. No food residue and reflux esophagitis were observed via endoscopic examination. Gallbladder contraction was observed in all the patients by ultrasonography after high-fat diet. Conclusion: Although long-term follow up and a larger number of patients are required to evaluate the functional outcomes, this new technique provides a minimally invasive surgical option for proximal early gastric cancer, especially in the cardiac area.
\end{abstract}

\section{Introduction}

The Japanese gastric cancer treatment guidelines (JGCG) defined standard gastrectomy as the adequate stomach resection and D2 lymph-node dissection. Due to the excellent long-term survival in early gastric cancer (EGC), function-preserving gastrectomy (FPG) was started to address the postoperative quality of life $(\mathrm{QOL})^{[1]}$. Apart from reducing the extent of gastrectomy, the surgeons aimed to preserve the pylorus and the perigastric vagus nerve during the surgical procedure. Proximal gastrectomy (PG) was labelled as FPG and preferred over total gastrectomy because it could mitigate the nutritional deterioration and weight loss associated with the latter. However, reflux esophagitis seriously affects the QOL, and no consensus exists on the reconstruction method to resolve this problem. Furthermore, conventional PG does not require vagus nerve preservation, which is precisely crucial in postoperative QOL. The hepatic branches could prevent postoperative gallstone formation and facilitate gastric motility ${ }^{[2]}$, and the celiac branches were related to the motility of the duodenum and the proximal jejunum and the regulation of gastrointestinal hormone secretion $^{[3]}$. In the present study, laparoscopic PG with vagus nerve preservation and double-flap technique (DFT) was successfully performed, representing a novel technique for proximal EGC. The preliminary results and technical aspects of this novel surgical technique were discussed.

\section{Materials and methods}

\section{Patients}

From March to May 2020, six patients underwent laparoscopic PG with vagus nerve preservation and doubleflap technique. This study was performed in line with the principles of the Declaration of Helsinki. Approval 
was granted by the ethics committee of our hospital. Informed consent was obtained from all individual participants included in the study. The clinical data of the patients were outlined in Table 1. Indications for this surgery included the tumor being located in the upper third of the stomach without esophageal invasion, the depth of tumor invasion confined to T1, no lymph node involvement, and lesions that could not be treated by endoscopic mucosal resection or local resection. The six-month follow-up visit involved a postoperative interview regarding gastroesophageal reflux disease and patient satisfaction. All patients received endoscopic and ultrasonic examination 6 months later.

\section{Vagus nerves preservation}

The patients were administered with general anaesthesia and placed in a supine position. The surgeon and assistant were positioned on the right and left side of the patient; the scopist was positioned between the patient's legs. After pneumoperitoneum was established at the umbilicus, a 2D laparoscope was inserted via this port with four ( 5 or $12 \mathrm{~mm}$ ) operating ports placed on the two sides of the upper abdomen. Firstly, the omentum was dissected from the mesocolon around the transition zone of lymph node station $4 \mathrm{~d}$ whilst preserving the right gastroepiploic vessels. Any posterior adhesion of the stomach was dissected. Next, the retroperitoneum was proximally dissected away along the spleen until the left gastroepiploic vessels were identified and they were then divided using hemoclips. The short gastric arteries were dissected close to the upper pole of the spleen. Lymph nodes stations 11d and 10 were not routinely dissected. Then, the gastric fundus was isolated by separating the gastrodiaphragmatic ligament. After the gastrohepatic ligament was fenestrated via an upper midline incision, the hepatic branches bifurcating from the anterior trunk of the vagus nerve were identified and fastened to protect them from damage (Figure 1a). The anterior gastric branches were identified and dissected by following the hepatic branches of the vagus nerve to the proximal side. Afterwards, the second branch of the right gastric artery free from any surrounding fat tissue along the lesser curvature was dissected to allow the lymph node along the lesser curvature to be dissected (Figure 1b). The surrounding tissue of the abdominal oesophagus was shifted to the actinal side, exposing the front of the abdominal oesophagus (Figure 1c). After the retroperitoneum was dissected, the anterior surface of the right crus of the diaphragm was opened and the trunk of the posterior vagus nerve was taped by exfoliating the oesophagus. The posterior gastric branches were identified and dissected by following the posterior vagus nerve (Figure 1d). The celiac branches splitting from the posterior trunk approached the left gastric artery as it neared the celiac ganglion. The left gastric vein was then clipped and divided. The left gastric artery was clipped and divided at the point distal to the celiac branches (Figure 1e). The retraction of the posterior trunk of the vagus nerve toward the surgeon enabled the lymph node dissection along the left gastric artery (Figure 1f). Dissection proceeded across lymph node stations 8a and 9 and continued along the spleen and the posterior gastric artery was cut. Finally, the exposed oesophagus was transected with a $60 \mathrm{~mm}$ Endo-Gia endoscopic linear stapler. The resected stomach was withdrawn from a $5 \mathrm{~cm}$-small incision in the upper abdomen. The surgeon observed the lesions and transected the stomach with a linear stapler whilst maintaining an adequate surgical margin and retaining two-thirds of the stomach.

\section{DFT}

An H-shaped $2.5 \times 3.5 \mathrm{~cm}$ seromuscular double-flap was created on the anterior wall of the remnant stomach through the auxiliary incision, leaving a region $1-2 \mathrm{~cm}$ from the proximal resection stump. The connective tissue between the muscle and the mucosa was cut under appropriate tension (Figure 2a). The gastric mucosa was opened for the anastomosis, keeping a $1 \mathrm{~cm}$ gap from the lower end of the flap (Figure $2 \mathrm{~b}$ ). Anastomotic stricture was prevented by keeping this gap slightly greater than the width of the oesophagus. Three-point sutures were used to fix the posterior side of the oesophagus to the remaining stomach at the upper edge of the flap (Figure 2c). Hand sewing method was used to perform the anastomosis of the oesophagus and the remnant stomach by using a V-Loc suture (Figure 2d). A single-layer continuous suture we used between all layers of the oesophagus and gastric mucosa for the posterior wall. A layer-to-layer suture was used for the anterior wall, where the first continuous suture was placed between the oesophagus and the stomach mucosa and the second interrupted suture was placed between the seromuscular layer of the stomach and the muscular layer of the oesophagus (Figure 2e). Finally, the flap was positioned to cover the anastomosis 
site in a Y-shape, with the midline first anchored for the flap to cover the widest possible area (Figure 2f).

\section{Results}

This surgical technique was performed in six patients, with no conversions to open surgery. The mean operative time was 270 minutes (range of 245-320 minutes) and the mean blood loss was $130 \mathrm{ml}$ (range of $40-210 \mathrm{ml}$ ). All the patients were started on a liquid diet on the second postoperative day. The X-rays were taken one week after the surgery and the results revealed no leakage. The contrast media passed smoothly through the anastomosis into the duodenum, resulting in no marked regurgitation into the oesophagus. No remarkable postoperative complications occurred. The mean postoperative hospital stay was 10.3 days (range of 8-13 days). No patients showed diarrhea and appetite loss, and body weight regained preoperative level 6 month later. No food residue and reflux esophagitis were observed via endoscopic examination. Gallbladder contraction was observed in all the patients by ultrasonography after high-fat diet. The rate of patient satisfaction was determined by direct interaction with the patients and found to be $100 \%$.

\section{Discussion}

The research focus of proximal EGC has shifted from improving prognosis to maintaining postoperative QOL, and reflux esophagitis after PG is an inevitable problem, which adversely affects the QOL. However, no consensus currently exists on the reconstruction method after PG. Esophagogastrostomy requires a single anastomosis and conforms to a physiological structure. Kamikawa described an anti-reflux procedure that was based on esophagogastrostomy, also called DFT ${ }^{[4]}$. The critical parts of this reconstruction involved the creation of a seromuscular double-flap and the implanted length of the oesophagus, which caused oesophageal reflux symptoms as the flap provides a one-way valve function. Additionally, vagus nerve preservation is also crucial to maintain postoperative QOL, which is usually resected during radical gastrectomy, thereby causing post-gastrectomy symptoms, such as diarrhoea, delayed gastric emptying and gallstone. Vagus nerve preservation is theoretically more meaningful in PG; however, vagus nerve preservation is not required in the surgical procedure. Only a limited number of studies was designed to investigate the value of vagus nerve preservation in PG with lower oesophageal sphincter preserved ${ }^{[5,6]}$. In fact, the lower oesophageal sphincter resection is inevitable in most cases under the premise of ensuring a sufficient margin.

Considering the role of gastric motility and cardiac sphincter in anti-reflux, laparoscopic PG with vagus nerve preservation and DFT was performed in the present study. The hepatic branches are very thin in most cases, which are usually resected inadvertently during surgery. Laparoscopic view allows magnified visualisation, making this delicate operation easier to perform with accuracy than open surgery. The celiac branches splitting from the posterior trunk approached the left gastric artery, which was clipped and divided at the point distal to the celiac branches. Retraction of the posterior trunk of the vagus nerve toward the surgeon enabled the lymph node dissection along the left gastric artery. This surgical technique was also performed in a patient with gastrointestinal stromal tumour located on the cardia and a patient with refractory gastroesophageal reflux disease. Intraoperative gastroscopy was not routinely performed and none of the patients developed anastomotic stricture. The six-month follow-up visit showed that the gallbladder maintained the normal concentration function and no reflux esophagitis were observed via endoscopic examination. No patients showed diarrhea and appetite loss, and body weight regained preoperative level.

Although long-term follow up and a larger number of patients are required to evaluate the functional outcomes, this new technique provides a minimally invasive surgical option for proximal EGC, especially in the cardiac area.

\section{Reference}

[1] Nomura E, Okajima K. Function-preserving gastrectomy for gastric cancer in Japan. World J Gastroenterol 2016; 22(26): 5888-5895.

[2] Wang CJ, Kong SH, Park JH, et al. Preservation of hepatic branch of the vagus nerve reduces the risk of gallstone formation after gastrectomy. Gastric Cancer 2021; 24(1): 232-244. 
[3] Kong SH, Kim SM, Kim DG, et al. Intraoperative Neurophysiologic Testing of the Perigastric Vagus Nerve Branches to Evaluate Viability and Signals along Nerve Pathways during Gastrectomy. J Gastric Cancer 2019; 19(1): 49-61.

[4] Kuroda S, Nishizaki M, Kikuchi S, et al. Double-Flap Technique as an Antireflux Procedure in Esophagogastrostomy after Proximal Gastrectomy. J Am Coll Surg 2016; 223(2): e7-e13.

[5] Matsumoto H, Murakami H, Kubota H, et al. Clinical outcome of lower esophageal sphincter- and vagusnerve-preserving partial cardiectomy for early gastric cancer of the subcardia. Gastric Cancer 2015; 18(3): 669-74.

[6] Hirai T, Matsumoto H, Iki K, et al. Lower esophageal sphincter- and vagus-preserving proximal partial gastrectomy for early cancer of the gastric cardia. Surg Today 2006; 36(10): 874-8.

Table 1 Clinical data of the six patients with proximal gastric cancer

\begin{tabular}{|c|c|c|c|c|c|c|c|}
\hline Case & Age & Sex & $\begin{array}{l}\text { Tumor size } \\
(\mathrm{mm})\end{array}$ & $\begin{array}{l}\text { Pathological } \\
\text { findings }\end{array}$ & $\begin{array}{l}\mathrm{PM} \times \mathrm{DM} \\
(\mathrm{mm})\end{array}$ & $\begin{array}{l}\text { Histologic } \\
\text { differentiation }\end{array}$ & $\begin{array}{l}\text { Lauren's } \\
\text { classification }\end{array}$ \\
\hline 1 & 58 & $\mathrm{M}$ & $15 \times 10$ & $\begin{array}{l}\text { pT1b } \\
(\mathrm{sm}), \mathrm{pN} 0 \\
(0 / 26)\end{array}$ & $15 \times 25$ & Undifferentiate & $\begin{array}{l}\text { Intestinal } \\
\text { type }\end{array}$ \\
\hline 2 & 70 & M & $35 \times 25$ & $\begin{array}{l}\text { pT1a }(\mathrm{m}), \\
\text { pN0 } \\
(0 / 32)\end{array}$ & $20 \times 20$ & Moderately & $\begin{array}{l}\text { Intestinal } \\
\text { type }\end{array}$ \\
\hline 3 & 75 & M & $10 \times 10$ & $\begin{array}{l}\text { pT1b } \\
(\mathrm{sm}), \mathrm{pN} 0 \\
(0 / 20)\end{array}$ & $40 \times 35$ & Poorly & $\begin{array}{l}\text { Diffuse } \\
\text { type }\end{array}$ \\
\hline 4 & 67 & M & $5 \times 3$ & $\begin{array}{l}\text { pT2 (mp), } \\
\text { pN0 } \\
(0 / 18)\end{array}$ & $25 \times 30$ & Moderately & $\begin{array}{l}\text { Diffuse } \\
\text { type }\end{array}$ \\
\hline 5 & 63 & M & $22 \times 15$ & $\begin{array}{l}\text { pT1b } \\
(\mathrm{sm}), \mathrm{pN} 0 \\
(0 / 28)\end{array}$ & $20 \times 40$ & Moderately & $\begin{array}{l}\text { Intestinal } \\
\text { type }\end{array}$ \\
\hline 6 & 71 & M & $5 \times 5$ & $\begin{array}{l}\text { pT1a }(\mathrm{m}), \\
\text { pN0 } \\
(0 / 24)\end{array}$ & $20 \times 30$ & Poorly & $\begin{array}{l}\text { Intestinal } \\
\text { type }\end{array}$ \\
\hline
\end{tabular}

$\mathrm{m}$, invasion of mucosa; sm, invasion of submucosa; mp, invasion of muscularis propria. PM, proximal margin; DM, distal margin

Figure 1 Preservation of the hepatic branches and the celiac branches.

Figure 2 Double-flap technique of the oesophagus and the gastric remnant. 

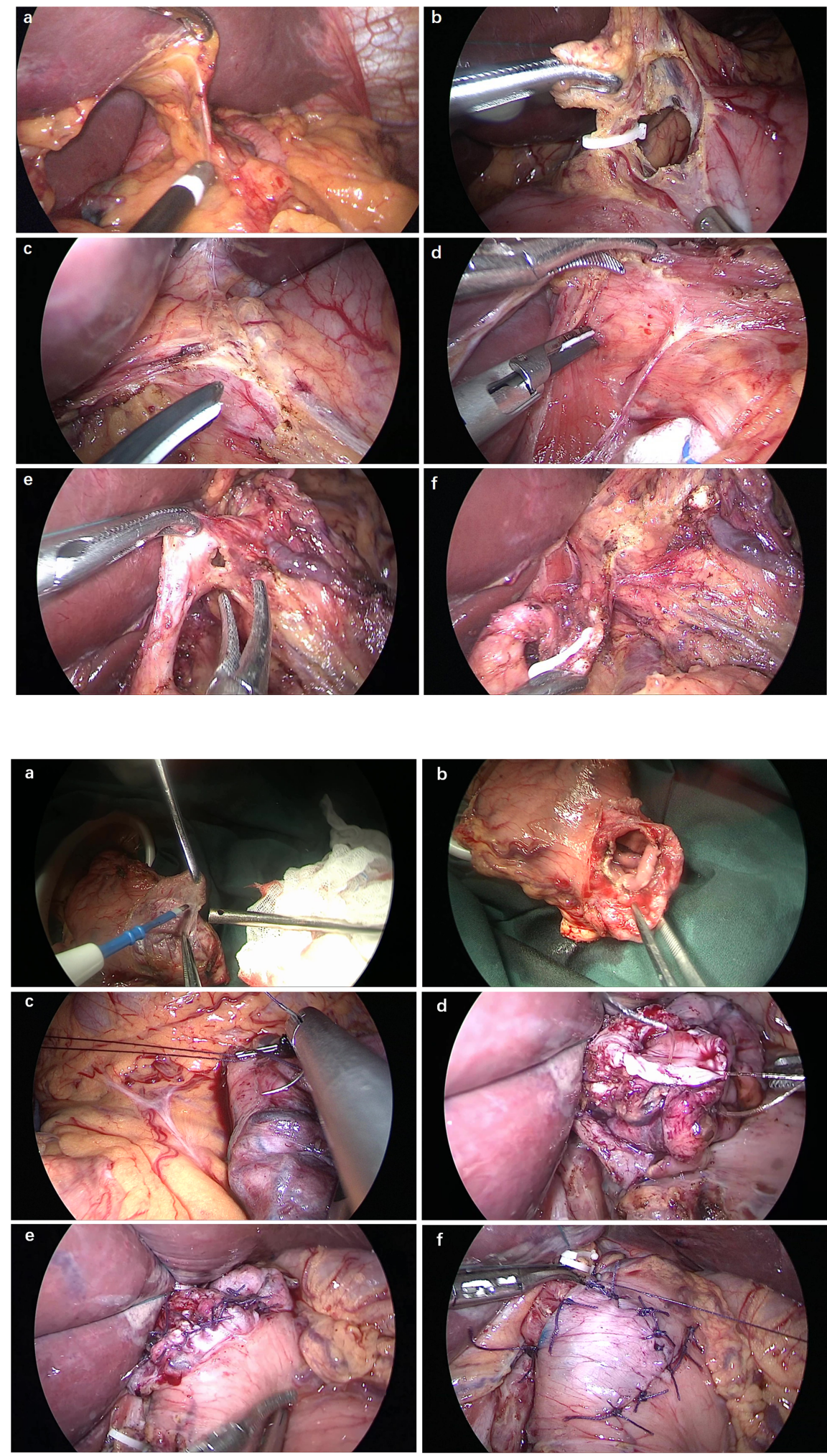

Hosted file 
Table 1.pdf available at https://authorea.com/users/412555/articles/521222-the-feasibilityof-vagus-nerve-preservation-and-double-flap-technique-in-laparoscopic-proximalgastrectomy 